\section{A trend analysis program for a repeated measures factor with equal or unequal intervals}

\author{
RICHARD F. ANTONAK and JAMES J. ROBERGE \\ Temple University. Philadelphia. Pennsylvania 19122
}

In many behavioral science experiments. the same subjects are exposed to all levels of a treatment (e.g., hours of sleep deprivation, dosage of a drug. or delay of reinforcement) that represents quantitative steps along an underlying continuum. Hence, additional information about the nature of the relationship between the repeated measures factor (treatment levels) and the dependent variable can be obtained by partitioning the treatment variation into nonoverlapping trend components (i.e., linear, quadratic. cubic. etc.) through the use of orthogonal polynomials. If the intervals of the repeated measures factor are equal, the computation of the trend analysis is facilitated by the use of the tabled orthogonal coefficients found in the standard statistical texts used by behavioral scientists (e.g., Kirk, 1968, p. 538; Winer, 1971. p. 878). On the other hand, when the intervals are unequal, the use of these tabled coefficients is inappropriate (cf. Roeckelein, 1971). However. Gaito (1965) has presented a general technique for generating the appropriate orthogonal coefficients for the unequal intervals case. This technique can also be employed to generate the tabled coefticients when the intervals are equal.

The program discussed in this paper performs a trend analysis on repeated measures factors of the types described above.

Input. The job deck consists of the following cards: a problem card, a levels format card, a levels card, label cards. a data format card, the data deck, and a last card. The problem card indicates the number of levels for each factor, the number of subjects per cell, whether a trend analysis is desired, and whether the repeated measures factor has equal or unequal intervals. The levels format card (for unequal intervals) is an F-type variable format card which indicates the location of the level magnitudes on the accompanying levels card. The label cards (one per factor) contain the alphanumeric labels for the factors. The data format card is an F-type variable format card which indicates the location of the raw scores on the subsequent data cards. Each of the data cards must contain the data for one subject; however, the use of more than one data card per subject is permitted. Finally, if the user wishes to terminate the program, the last card must have the word FINISH punched in Columns 1-6. On the other hand, if the user wishes to analyze another set of data, the last card is a blank card and the job deck is arranged sequentially (as described above), beginning with the problen card.

Output. The printed output includes (a) the labels for the factors, (b) an analysis of variance summary table (i.e., sources of variation. sums of squares, degrees of freedom, mean squares. and $F$ ratios) which is presented in a form similar to that used by Winer (1971. p. 592), and (c) matrices of means, standard deviations, and standard errors, for all main effects and interactions. Furthermore, if the user chooses to have a trend analysis performed on the repeated measures factor. then the printed output also contains (a) the magnitudes of the levels assigned by the computer (for equal intervals) or specitied by the user (for unequal intervals), (b) the orthogonal coefficients. (c) checks of orthogonality (sums and cross-products), and (d) the trend components for the within-subjects main effect, interaction(s), and error variation.

Computer and language. The program is written in FORTRAN IV for processing by computers in the IBM 360 (or the CDC 6000) series. Well docunented, it has variable names that are mnemonic and correspond to the symbols used in Winer's (1971) computational formulas, to facilitate modification by users.

Restrictions. The progran has the following limitations: (a) the design can have either two or three factors with repeated measures on one of the factors. (b) each factor can have a maximum of 10 levels, (c) there cannot be more than 10.000 observations, and (d) there must be an equal number of observations in each cell.

Availability. Copies of this paper, test data. and a source listing. which includes printed output for sample problems for two- and three-factor experimental designs. may be obtained without charge by writing to James J. Roberge. Temple University, Department of Educational Psychology. Philadelphia, Pennsylvania 19122.

\section{REFERENCES}

Gaito. J. Unequal intervals and unequal $\mathrm{n}$ in trend analyses. Psychological Bulletin. 1965, 63, 125.127.

KIRK, R. E. Experimental design: Procedures for the behavioral sciences. Belmont, Calif: Brooks/Cole, 1968.

Roeckelein, J. E. Failure to make orthogonal polynomial adjustments for unequally spaced intervals yields inaccurate F values in trend tests. Perceptual and Motor Skills, 1971, 33, 1179-1183.

WINER, B. J. Statistical principles in experimental design (2nd ed.). New York: McGraw-Hill. 1971. 